\title{
Fatigue strength evaluation for individual strands of overhead conductors using a biaxial fretting fatigue test bench
}

\author{
Amine Omrani ${ }^{1}$, Sébastien Langlois ${ }^{1}$, Pierre Van Dyke ${ }^{2}$, Sasan Sattarpanah Karganroudi ${ }^{1}$, and Sébastien Lalonde \\ ${ }^{1}$ Université de Sherbrooke, Civil engineering Department, Canada \\ ${ }^{2}$ Institut de recherche d'Hydro-Québec, Performance, evolution and characterization of transmission line assets, Canada \\ ${ }^{3}$ Ecole de Technologie Supérieure, Mechanical engineering Department, Canada
}

\begin{abstract}
This paper presents a biaxial fretting fatigue test bench which provides the capability of performing fretting fatigue experiments on individual wires of a conductor combining the effect of both alternating tension and bending loadings to represent a more realistic state of a conductor individual strand under periodic loading caused by aeolian vibrations. Preliminary tests with only uniaxial alternating tension loading were carried out on 1350-H19 aluminum wires having the same mechanical and geometric characteristics as those of the ACSR Bersfort conductor aluminum strands. Different levels of alternating stress were tested in order to validate the performance of the apparatus. Preliminary results showed that the experimental setup allows reproducing the local loading state which leads to the fretting fatigue damage on the tested wires. Biaxial tests were also conducted at a high level of alternating loadings. The results of these tests reveals that, at high alternating stress amplitudes, the biaxial loading allows to observe some fretting fatigue failures, whereas early plain fatigue failures were observed when applying similar uniaxial loading.
\end{abstract}

\section{Introduction}

The knowledge of the performance over time of overhead conductors requires the creation of a series of tools to evaluate their fatigue strength. The complexity of the fretting fatigue problem, the main cause of the failure of these conductors, involves the use of experimental and numerical methods to analyze the phenomenon and to understand its mechanisms and effects. The method of fatigue testing of overhead conductors is well explained at the global scale from an experimental point of view in IEC 62568 international standard [1], however, fatigue test results are mainly available for ACSR conductors and there are only few results available for other conductor types [2]. The numerical model of a clamp/conductor system developed by LALONDE [3] takes into account inter-wire and clamp wire contacts in a stranded conductor and thus provides a more realistic description of the local loading state. Also, it has been shown, based on the experimental results of LÉVESQUE tests [4], that this numerical approach is capable of evaluating the state of local solicitation in terms of stress and strain at any point of the conductor, which consequently makes it possible to locate the critical zones that are susceptible to failure. At the local scale, experimental tests on individual wires are complementary to standard fatigue tests on conductors for characterization of fretting fatigue damages. Several research works brought back the experimental study to tests on individual strands in order to better evaluate the local effect of this phenomenon on wires. However, most of the available studies were carried out using uniaxial loading on the tested wires, in which some developed fretting fatigue test benches apply alternating tension [5] while others apply alternating bending [6]. LALONDE [3] has shown through a numerical analysis of stress distribution in a clamp/conductor assembly that conductor wires mainly sustain tension loads near the clamp exit but support larger bending stress in-between KE (Keeper Edge) and LPC (Last Point of Contact) positions (Fig.1). Therefore, the combined effect of these two loading parameters may need to be taken into consideration to better describe the stress state that leads to wires failure. 


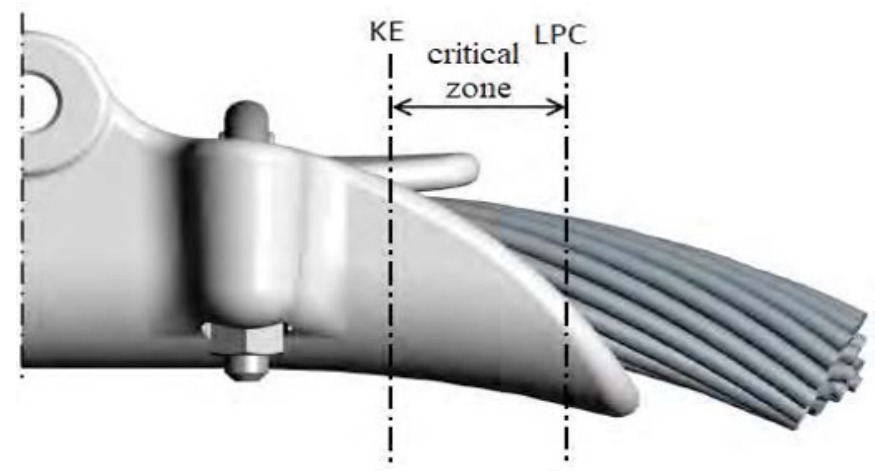

Fig.1. Critical zone for the fretting fatigue damage of overhead conductors near the suspension clamp (reproduced from LALONDE [7]).

The proposed device presented here is a biaxial fretting fatigue test bench combining the effect of both alternating tension and bending loadings, based on the exploratory experimental works of LALONDE [7]. Loading parameters used in the preliminary biaxial tests were determined from LALONDE [3] numerical model that reproduces the assembly of the ACSR Bersfort conductor with a standard metallic clamp. Starting from this model and considering the maximum level of the alternating stress in-between KE and LPC, the critical contact point that is the most susceptible to failure was identified and then the local loading state of this point was extracted. These loads can be classified into two main components: a static loading composed of a bending displacement, a tension force and a normal contact force, to which are added alternating loading amplitudes of tension force and bending displacement (Fig.2).

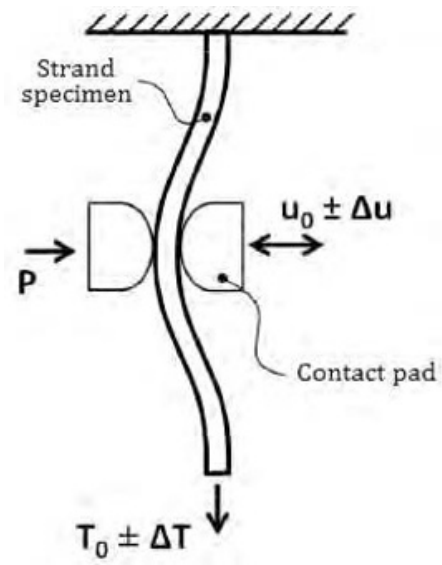

Fig.2. Loadings configuration on the biaxial fretting fatigue test bench (reproduced from LALONDE [7]).

Sections 2 and 3 of the paper provide, respectively, a detailed description of the developed test bench and the experimental procedure followed for carrying out the fretting fatigue tests. The configuration used for the preliminary tests is presented in section 4, while section 5 is devoted to the analysis and discussion of the obtained results.

\section{Experimental test bench}

\subsection{Test Bench Configuration}

The fretting fatigue test bench is designed to allow the application of a biaxial loading of tension and bending on the wire specimen. It is composed mainly of three hydraulic actuators equipped with a $5 \mathrm{kN}$ load cell each, fixed on a rigid metallic table and connected to a hydraulic pump (Fig.3). A servo-hydraulic test system capable of cyclic loading frequencies up to $100 \mathrm{~Hz}$ is used to control the actuators. 


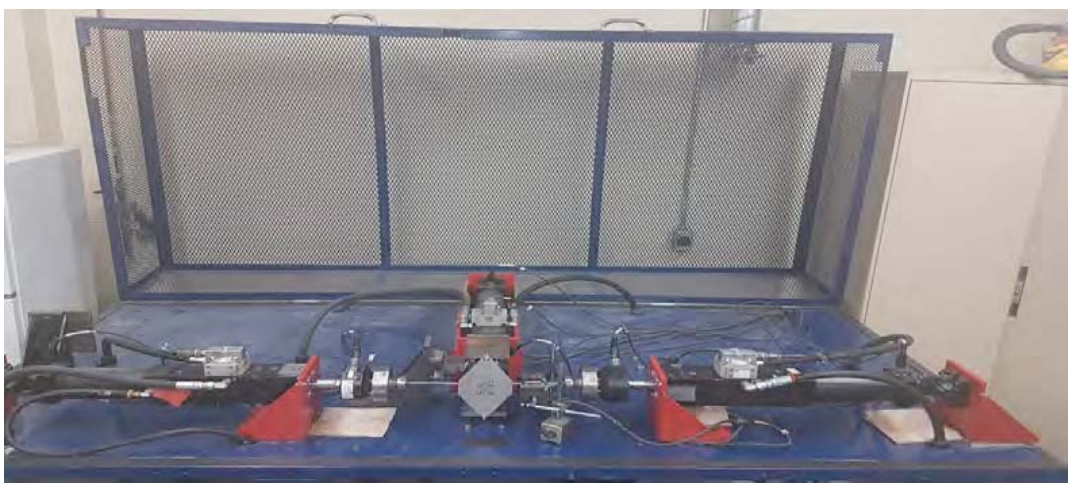

Fig.3. General overview of the experimental setup.

The aluminum wire specimen is attached to the axial actuators labeled as $A_{1}$ and $A_{2}$ by means of ER clamping collets as shown in Fig. 4. Both of these actuators are used to apply the tension force on the tested specimen, while the transverse actuator, labeled as $\mathrm{A}_{3}$, enables the application of the bending displacement. The local reproduction of the contact conditions is ensured through contact pads simulating the internal curvature radius of the suspension clamp. They are designed to be easily removable allowing the use of various pad geometries without major adjustments which makes it possible to reproduce a wide variety of fretting fatigue conditions involving those whom occur at the conductor-clamp and the inter-wire contact points. These pads, made of aluminum, are fabricated on a numerically controlled machine-tool and they are installed on the transversal actuator via a mounting bracket. The normal contact force is maintained constant throughout the test using a metallic plate system attached to the transverse actuator by means of two bolts.
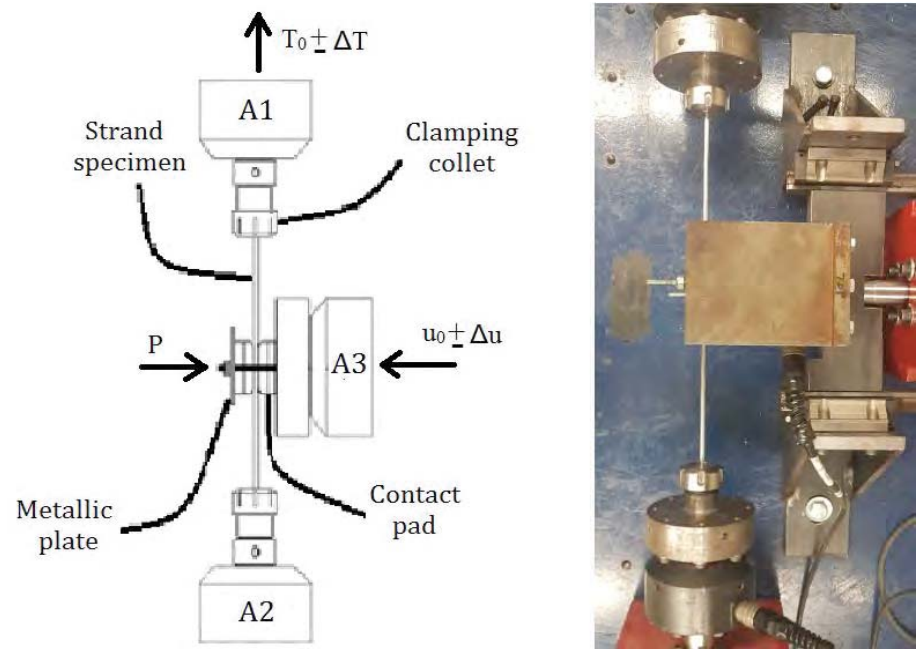

Fig.4. Fretting fatigue test bench composition.

\subsection{Data Acquisition}

Data acquisition is ensured through an MTS FlexTest Controller System that is connected to the three $5 \mathrm{kN}$ load cells. The master data acquisition program is controlled through the Station Manager window which has two counters, one for the number of cycles and the other for the time elapsed since the start of the test. From this main window, we can have access to six other windows to follow up the evolution of the various loading parameters during the test (Fig.5). The Manual Command, the Signal Auto Offset and the MPT Procedure Editors are control windows that allow respectively adjusting manually the position of the actuators in force and in displacement, to define their offsets and to enter and save the different parameters of the test. The Meters, the Scope and the Detectors are display windows which display respectively the instantaneous values of the displacements and the forces of each actuator, a real-time graphic of their evolution and the various errors that occur during the test. 


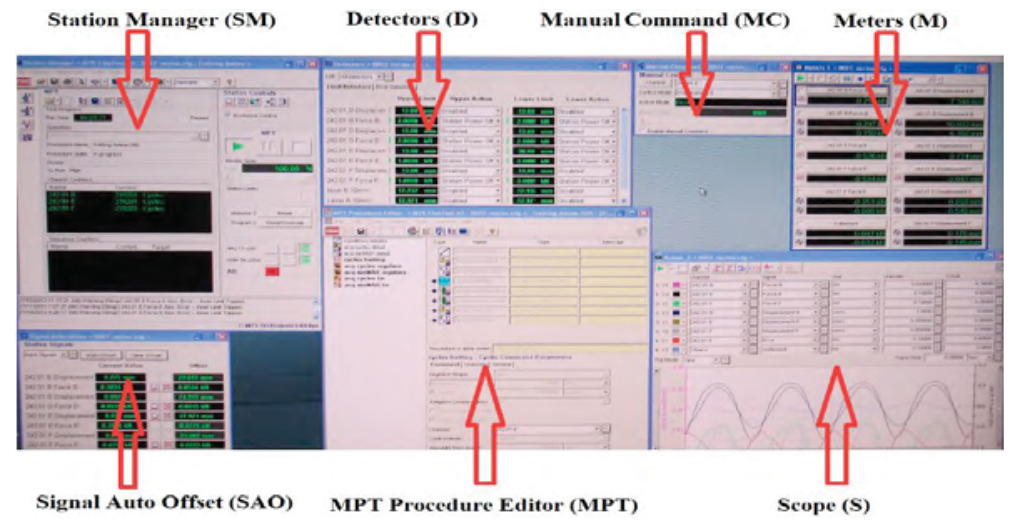

Fig.5. FlexTest controller system display.

The load cells installed in this test setup assure an accurate measurement of the tension forces at both ends of the wire specimen as well as the normal contact force in the transverse direction. In addition to the induced forces on the conductor wire, the instantaneous displacement of each actuator is recorded by the controller system. In the uniaxial tests, the tangential force $Q$ and the axial elongation $\delta$ which represents an estimation of the micro-sliding at the contact interface between the wire and the pads can be determined from these measurements as indicated in Eqs 1 and 2:

$$
\begin{gathered}
2 * Q \approx\left|T_{A 1}-T_{A 2}\right| \\
\delta \approx \frac{Q * L}{2 * E_{a} * S_{a}}
\end{gathered}
$$

Where $T_{A 1}$ and $T_{A 2}$ are the tension forces, respectively, at the axial actuators $\mathrm{A}_{1}$ and $\mathrm{A}_{2}$, and $L, E_{a}$ and $S_{a}$ are respectively the length of the specimen, its Young's modulus and its section area. In fact, due to the contact symmetry between the tested specimen and both pads, the tangential force $Q$ in each contact interface is evaluated to be the half of the force differential of both axial actuators $\mathrm{A}_{1}$ and $\mathrm{A}_{2}$. Consequently, the elongation $\delta$ is deduced using Hooke's law as shown in Eq. 3:

$$
\sigma=E_{a} * \varepsilon \longleftrightarrow \frac{Q}{S_{a}}=E_{a} * \frac{\delta}{L / 2}
$$

The measurement of these two parameters makes it possible to follow their evolution and to adjust them in order to induce the mixed fretting regime [8] corresponding to the most critical one that leads to the crack initiation and the damage of the strand under the imposed loading conditions [9]. In the biaxial tests, the control of these two parameters becomes a difficult task due to the bending effect induced by the transverse actuator $\mathrm{A}_{3}$ and therefore they are not evaluated in the present study for this test configuration.

\section{Experimental procedure}

The experimental procedure of carrying out the tests is almost the same for the uniaxial or the biaxial loading tests. It can be divided into two main phases: the phase of setting up the wire specimen under static loads and the phase of adjusting the experimental parameters to induce the alternating loads.

For the uniaxial tests, the first step is to fix both ends of the wire specimen at the actuators $A_{1}$ and $A_{2}$ so that the center of the wire coincides with the center of the contact pads as shown in Fig.4. Once the wire is fixed, the static tension $T_{0}$ is applied using the actuator $A_{1}$. The second step is to control the actuator $A_{3}$ in displacement until the contact pad attached to it comes into contact with the wire and so its force and displacement are set to zero. The third step consists of applying the normal contact force $P$ on the wire by clamping the second contact pad attached to the metallic plate. Before moving to the dynamic loading phase, the initial displacement of both actuators $\mathrm{A}_{1}$ and $\mathrm{A}_{2}$ must be set to zero.

After setting up the specimen and applying the static loads, the dynamic phase can be started. In this phase we proceed by entering the value of the alternating loading parameters on the MPT Procedure Editors such as the peak values of the tension force for the actuator $A_{1}\left(T_{0}+\Delta T\right.$ and $\left.T_{0^{-}} \Delta T\right)$, the peak values of the displacement for the actuator $\mathrm{A}_{2}$, the frequency and the duration limit of the test expressed in a maximum number of cycles after which the test will be automatically stopped. Once all parameters are saved, the test is run and the focus is on the adjustment of the displacement of the actuator $A_{2}$ by increasing or decreasing its peak values so that the right value is obtained for the tangential force $Q$. This parameter is determined by the value of $\mu P$, where $\mu$ is the static coefficient of friction, in order to cause the crack initiation. In summary, the actuator $A_{1}$ is controlled in force such that both the static and dynamic 
tension $\left(T_{0}+\Delta T\right.$ and $\left.T_{0^{-}} \Delta T\right)$ are applied, while the actuator $\mathrm{A}_{2}$ is controlled in displacement such that the tangential force corresponding to a state of fretting fatigue is obtained.

For the biaxial tests, the same steps described previously are followed. However, before applying the normal contact force, a static bending $u_{0}$ is applied onto the wire specimen via the transverse actuator $\mathrm{A}_{3}$. On the MPT Procedure Editors the peak values of the displacement for this actuator $\left(u_{0}+\Delta u\right.$ and $\left.u_{0}-\Delta u\right)$ are added and the phase shift between the tension force of $\mathrm{A}_{1}$ and the bending displacement of $\mathrm{A}_{3}$ is adjusted to get the maximum bending amplitude with the minimum tension variation and inversely. This adjustment is based on LALONDE numerical study [7] which revealed a $180^{\circ}$ phase shift between the variation of the tension and bending loads near the suspension clamp exit.

\section{Tests configuration}

Before starting the preliminary tests, it is important to get an estimation of the static coefficient of friction at the contact between the wire and the pads. This parameter was evaluated to be 0.43 using a simple test which consists in attaching a wire specimen on one side to an axial actuator, applying a clamping force to its center using the contact pads and pointing a laser to its unattached end to measure its axial displacement. A $5 \mathrm{~N} / \mathrm{s}$ increasing tension force is applied to the wire until it starts to slide which allows deducing the friction force at the contact area. As explained in the previous section, the evaluation of this parameter allows estimating the required tangential force $Q$ depending on the normal contact force $P$. All fretting fatigue tests were carried out on 1350-H19 aluminum wires cut and straightened manually from a coil of wire. The wire specimens have a length of $350 \mathrm{~mm}$ and a diameter equivalent to that of the ACSR Bersfort conductor aluminum strands $(d=4.24 \mathrm{~mm})$. If there was no failure after 20 million cycles, the test was stopped.

As mentioned earlier, two types of tests were done, uniaxial and biaxial loading tests. Table 1 presents the parameters of uniaxial tests. These tests were carried out at a frequency of $24 \mathrm{~Hz}$. Four levels of alternating stress $\sigma_{\mathrm{a}}$ and two values of the normal contact force $P$ were tested. Except for the higher level of alternating stress $\sigma_{a}=56 M P a$, the static tension $T_{0}$ was the same for all uniaxial tests. The value selected for $T_{0}$ is very close to the static tension obtained through the numerical model for biaxial tests. For $\sigma_{a}=56 \mathrm{MPa}, T_{0}$ was higher to avoid the compression of the tested specimen.

Table 1. Uniaxial tests configuration.

\begin{tabular}{|c|c|c|c|c|c|}
\hline$\sigma_{\mathrm{a}}(\mathrm{MPa})$ & $\Delta \mathrm{T}(\mathrm{N})$ & $\mathrm{P}(\mathrm{N})$ & $\mathrm{T}_{0}(\mathrm{~N})$ & Frequency $(\mathrm{Hz})$ & Test number \\
\hline \pm 56 & \pm 791 & 240 & 850 & \multirow{6}{*}{24} & $1 ; 2 ; 3$ \\
\hline \multirow{2}{*}{ \pm 43} & \multirow{2}{*}{ \pm 607} & 240 & \multirow{5}{*}{640} & & $4 ; 5$ \\
\hline & & 100 & & & $6 ; 7$ \\
\hline \pm 35 & \pm 494 & 240 & & & $8 ; 9 ; 10$ \\
\hline \multirow{2}{*}{ \pm 25} & \multirow{2}{*}{ \pm 353} & 240 & & & 11 \\
\hline & & 100 & & & $12 ; 13$ \\
\hline
\end{tabular}

The loads implemented for the biaxial tests were extracted from the numerical model of LALONDE [3] which simulates the assembly of an ACSR Bersfort conductor with a standard metallic clamp submitted to a peak-to-peak bending amplitude $Y_{b}=0.75 \mathrm{~mm}$ measured at $89 \mathrm{~mm}$ from LPC. For reasons of stability of the command signals and to reduce the effect of the dynamic acceleration due to the bending displacement of the actuator $\mathrm{A}_{3}$, these tests were done at a lower frequency of $12 \mathrm{~Hz}$ compared to the uniaxial ones. Table 2 illustrates the values of the different parameters used in this type of tests.

Table 2. Biaxial tests configuration.

\begin{tabular}{|c|c|c|c|c|c|c|c|}
\hline$\sigma_{\mathrm{a}}(\mathrm{MPa})$ & $\Delta \mathrm{T}(\mathrm{N})$ & $\mathrm{P}(\mathrm{N})$ & $\mathrm{T}_{0}(\mathrm{~N})$ & $\mathrm{u}(\mathrm{mm})$ & $\Delta \mathrm{u}(\mathrm{mm})$ & Frequency $(\mathrm{Hz})$ & Test number \\
\hline \pm 56 & \pm 274 & 240 & 630 & 8,13 & $\pm 1,95$ & 12 & $14 \rightarrow 18$ \\
\hline
\end{tabular}

\section{Results and discussion}

\subsection{General observations}

The first challenge encountered in the calibration of the experimental setup was to find the right amplitude of the micro displacement of the actuator $\mathrm{A}_{2}$ which would lead to a tangential force $Q \approx \mu P$ in order to establish the mixed stick-slip 
condition, most propitious to crack initiation and consequently the break of the tested wire under fretting fatigue [8]. A micrograph of the resulting fretting mark of both uniaxial and biaxial tests is shown in Fig. 6. In both cases, the fretting fatigue crack was initiated in the contact area. A clear mark is observed in the center of the contact area where no relative motion between wire and pads would occur, surrounded by a dark mark representing the micro slip area. This observation is clearer on the uniaxial tested wires than on the biaxial ones and that can be explained by the effect of the alternating bending in biaxial tests which expends the contact area and consequently enlarges the slipping mark to the detriment of the sticking mark. The alternating bending effect is also noticed by comparing the geometry of the contact area in both types of tests. It can be seen that the contact area in the uniaxial tests has an elliptical shape while in the biaxial ones it is almost linear and more extended.

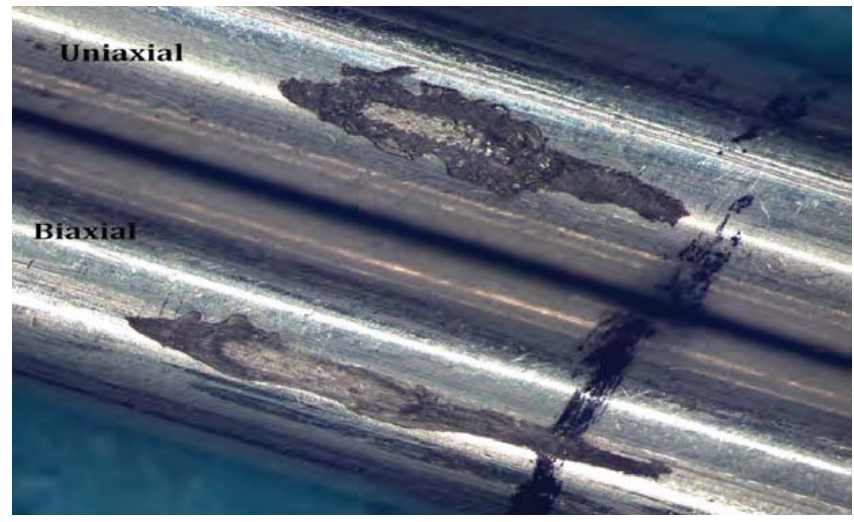

Fig.6. Fretting fatigue marks under uniaxial and biaxial loading.
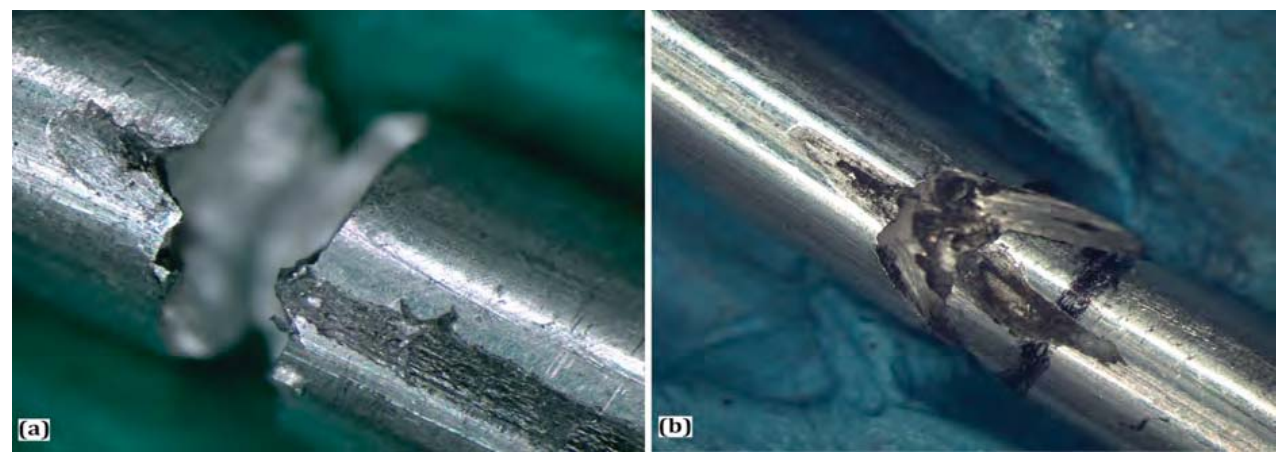

Fig.7. Fretting fatigue breaks in (a) biaxial and (b) uniaxial test.

Fig. 7 is a micrograph of fretting breaks that occurred respectively in a biaxial and a uniaxial test. In the first case, the breaking point is near the last point of contact while in the second one, it is virtually located in the center of the contact area. When comparing the cracking plan of both test types, we notice two different fracture patterns. For the uniaxial tests, the cracking plane is inclined about $45^{\circ}$ to the wire axis which confirms the presence of the mixed stickslip condition. However, in the case of the biaxial tests, the break occurs along a plane parallel to the section of the wire thus proving the dominance of the partial slip regime [10]. In fact, the tangential force can be easily controlled in the uniaxial tests to establish the mixed fretting regime, which is not the case for the biaxial ones where the alternating bending induces an additional component in the tangential force which is difficult to control.

\subsection{Uniaxial tests results}

The results of the uniaxial tests are presented in table 3. During these tests, the estimated micro-sliding was always in the range of 5 to $50 \mu \mathrm{m}$, the typical values resulting in fretting fatigue [11]. At a very high alternating stress level $\left(\sigma_{a}= \pm 56 \mathrm{MPa}\right)$, all recorded breaks were due to plain fatigue and were located outside the contact area, at the attachment point of the wire to the actuator $\mathrm{A}_{2}$. However, at moderate levels of the alternating stress $\left(\sigma_{a}= \pm 43 M P a\right.$ and $\sigma_{a}= \pm 35 \mathrm{MPa}$ ), most of the breaks were in the contact area between the wire and the pads and were caused by fretting fatigue. At the lowest alternating stress level $\left(\sigma_{a}= \pm 25 \mathrm{MPa}\right)$, tests reached the fixed lifetime limit without recording a 
break. The fatigue life of the wire is slightly affected by the normal contact force. At high amplitude, the decrease of this force results in a decrease in the number of cycles to failure, whereas near the endurance limit the variation of this force has practically no effect on the fatigue life.

Table 3. Results of the uniaxial tests.

\begin{tabular}{|c|c|c|c|c|c|}
\hline Test number & Number of cycles & Breaking position & Breaking cause & $\mathrm{Q}(\mathrm{N})$ & $\delta(\mu \mathrm{m})$ \\
\hline 1 & 1.760 .991 & At the attachment point $\mathrm{A}_{2}$ & Plain fatigue & 90 & 16 \\
\hline 2 & 1.439 .617 & At the attachment point $\mathrm{A}_{2}$ & Plain fatigue & 95 & 17 \\
\hline 3 & 735.500 & At the attachment point $\mathrm{A}_{2}$ & Plain fatigue & 93 & 17 \\
\hline 4 & 3.209 .610 & In the contact area & Fretting fatigue & 92 & 16 \\
\hline 5 & 7.544 .760 & At the attachment point $\mathrm{A}_{2}$ & Plain fatigue & 95 & 17 \\
\hline 6 & 2.634 .397 & In the contact area & Fretting fatigue & 42 & 8 \\
\hline 7 & 2.418 .054 & In the contact area & Fretting fatigue & 50 & 9 \\
\hline 8 & 6.933 .267 & In the contact area & Fretting fatigue & 112 & 20 \\
\hline 9 & 7.143 .537 & At the attachment point $\mathrm{A}_{2}$ & Plain fatigue & 107 & 19 \\
\hline 10 & 7.356 .070 & In the contact area & Fretting fatigue & 112 & 20 \\
\hline 11 & 20.000 .000 & No break & ESIIIIIIIIIIIIII & 109 & 20 \\
\hline 12 & 20.000 .000 & No break & enIIIIIIIIIIIII & 40 & 7 \\
\hline 13 & 20.000 .000 & No break & 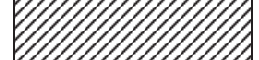 & 32 & 6 \\
\hline
\end{tabular}

\subsection{Biaxial tests results}

Biaxial tests were also performed for the loading configuration identified in table 2 earlier. As presented in table 4 , the majority of the breaks were due to fretting fatigue and were in the contact area between the tested specimen and the pads. Fig.8 illustrates the results of both uniaxial and biaxial tests as S-N curves. At this stage of the experimental study, the results obtained are not sufficient to fully compare both types of tests in terms of fatigue life. However, it can be observed that the setup allows getting some fretting fatigue failures at high stress amplitudes for biaxial loading, whereas early plain fatigue failures are recorded when performing similar uniaxial tests. Furthermore, the relation between the number of cycles to failure and the alternating stress appears to be different for the uniaxial and biaxial tests.

Table 4. Results of the biaxial tests.

\begin{tabular}{|c|c|l|l|}
\hline Test number & Number of cycles & \multicolumn{1}{|c|}{ Breaking position } & Breaking cause \\
\hline 14 & 3.845 .950 & In the contact area & Fretting fatigue \\
\hline 15 & 2.910 .136 & In the contact area & Fretting fatigue \\
\hline 16 & 3.112 .426 & In the contact area & Fretting fatigue \\
\hline 17 & 4.092 .373 & In the contact area & Fretting fatigue \\
\hline 18 & 7.449 .855 & At the attachment point $\mathrm{A}_{2}$ & Plain fatigue \\
\hline
\end{tabular}




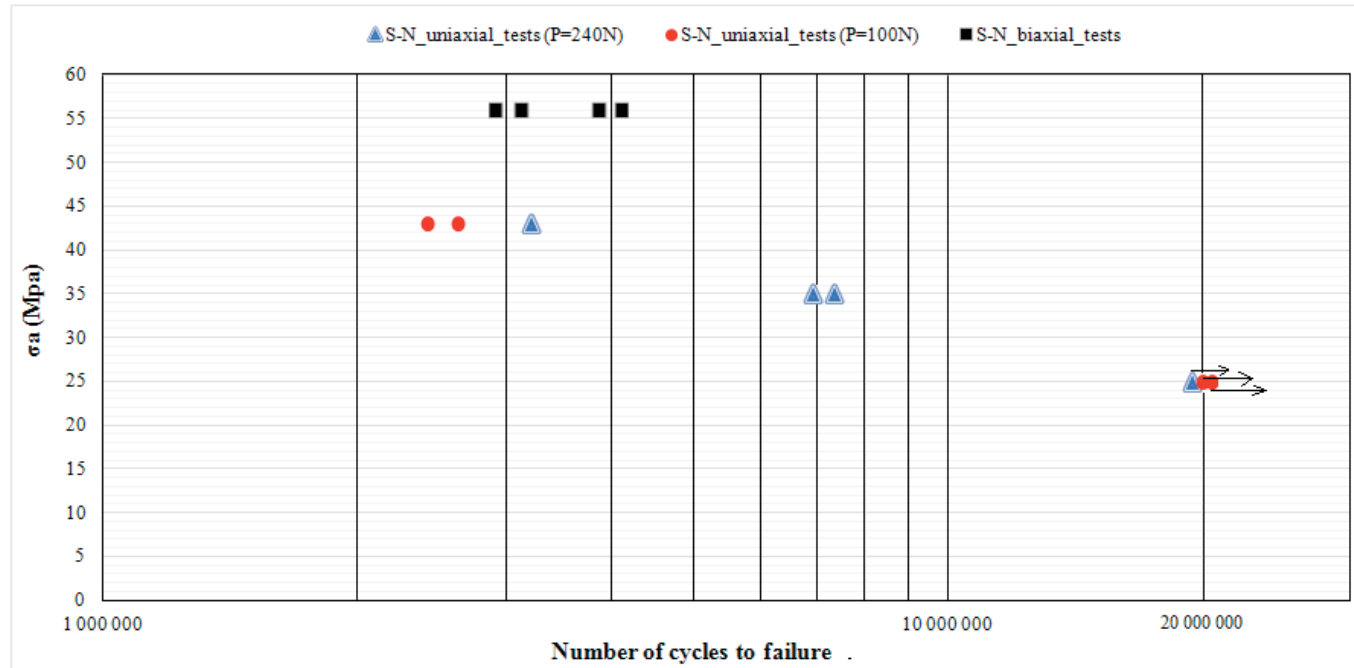

Fig. 8. Fatigue data of the uniaxial and the biaxial tests.

\section{Conclusions}

The design of a biaxial fretting fatigue test bench has been presented in this paper. The results of the preliminary tests have shown that this experimental setup allows performing both uniaxial and biaxial loading tests. At very high levels of alternating stress, the developed test bench enables to perform biaxial tests better than uniaxial ones. The performance of the developed device has been verified through the creation of fretting marks leading to fretting fatigue failure. Some breaks have occurred outside the contact area, at the attachment point of the wire to the axial actuator, which requires improving the clamping system in order to minimize the stress level at both ends of the tested wires. A wide variety of fretting fatigue tests representing strand damages occurring at the conductor-clamp and the inter-wire contact points and involving different parameters such as the normal contact force, the alternating tension and the alternating bending can be reproduced in the presented test bench.

\section{References}

1. IEC 62568 International Standard, Overhead lines - Method for fatigue testing of conductors (2015)

2. L. Cloutier, S. Goudreau, A. Cardou, EPRI Transmission Line Reference Book - Wind Induced Conductor Motion, 3.1-3.56 (2006)

3. S. Lalonde, R. Guilbault, S. Langlois, Numerical analysis of ACSR conductor-clamp systems undergoing windinduced cyclic loads, IEEE Trans. on Pow. Deli., 33: 1518-1526 (2018)

4. F. Lévesque, S. Goudreau, A. Cardou, L. Cloutier, Strain measurements on ACSR conductors during fatigue tests IExperimental method and data, IEEE Trans. on Pow. Deli., 25: 2825-2834 (2010)

5. P. U. Wittkowsky, P. R. Birch, J. Dominguez, S. Suresh, An apparatus for quantitative fretting fatigue testing, Fatigue Fract. Eng. Mater. Struct., 22: 307-320 (1999)

6. J. Lanteigne, L. Cloutier, A. Cardou, Fatigue life of aluminum wires in all aluminum and ACSR conductors, CEA, Canada (1986)

7. S. Lalonde, Stratégie de modélisation 3D des solides toronnés appliquée à l'étude de la fatigue des conducteurs de lignes de transport d'énergie électrique, Université de Sherbrooke, Canada (2017)

8. O. Vingsbo, S. Söderberg, On fretting maps, Wear, 126: 131-147 (1988)

9. Z. R. Zhou, L. Vincent, Mixed fretting regime, Wear, 181-183: 531-536 (1995)

10. Z. R. Zhou, A. Cardou, S. Goudreau, M. Fiset, Fundamental investigations of electrical conductor fretting fatigue, Trib. Inter., 29: 221-232 (1996)

11. S. Suresh, Fatigue of materials, 679 (1998). 